\title{
Germanica
}

\section{La crise économique au théâtre. L'esthétique du transitoire et le néoréalisme dramatique (Zeller, Marks, Berthiaume)}

\section{Hanna Klessinger}

\section{(Q) OpenEdition}

Journals

Édition électronique

URL : https://journals.openedition.org/germanica/8178

DOI : 10.4000/germanica. 8178

ISSN : 2107-0784

\section{Éditeur}

Université de Lille

\section{Édition imprimée}

Date de publication : 1 décembre 2019

Pagination : $169-178$

ISBN : 978-2-913857-44-5

ISSN : 0984-2632

\section{Référence électronique}

Hanna Klessinger, « La crise économique au théâtre. L'esthétique du transitoire et le néoréalisme dramatique (Zeller, Marks, Berthiaume) », Germanica [En ligne], 65 | 2019, mis en ligne le 01 janvier 2021, consulté le 08 janvier 2022. URL : http://journals.openedition.org/germanica/8178; DOI :

https://doi.org/10.4000/germanica.8178 


\title{
(Re)lectures
}

\author{
La crise économique au théâtre. \\ L'esthétique du transitoire et le néoréalisme \\ dramatique (Zeller, Marks, Berthiaume)
}

Hanna Klessinger
Freiburg im Breisgau

Comment le théâtre d'aujourd'hui répond-il au sentiment diffus de crise économique et sociale globalisée qui a envahi la société contemporaine ? En d'autres termes, certaines de ses formes actuelles peuventelles - en fonction du contexte ou selon le regard qu'on porte sur elles être considérées comme une réponse esthétique adéquate à la crise ? C'est dans cette perspective que nous analyserons des pièces de théâtre contemporaines dans leur rapport au transitoire et au provisoire, dans la mesure, en effet, où le sentiment d'instabilité ou d'insécurité profondes face à la crise se reflète dans une « esthétique du transitoire ».

Trois pièces de trois jeunes dramaturges de langue allemande, anglaise et française seront revisitées dans une perspective comparatiste : X-Freunde de l'Allemande Felicia Zeller, Bethany de l'Américaine Laura Marks, et enfin, Yukonstyle de la Québécoise Sarah Berthiaume. Ces pièces datant des années 2012 et 2013 figurent ici - d'un point de vue thématique aussi bien que formel - comme paradigme de cette nouvelle esthétique du transitoire. En même temps, elles suivent une tendance dans le théâtre contemporain que l'on peut qualifier de néoréaliste. Ce « nouveau réalisme » ou « néoréalisme » succède à des tendances dites 
«postdramatiques $»^{1}$. Même si des oppositions aussi strictes ne sont pas toujours faciles à défendre, ces termes décrivent malgré tout une tendance de fond : le retour aux sujets 'réels', aux dialogues, aux personnages dramatiques et aux intrigues. L'analyse qui suit cherche donc également à montrer une correspondance entre l'esthétique du transitoire et le néoréalisme.

Avant d'interroger les trois pièces, il sera brièvement question du contexte social et politique qui sous-tend cette esthétique du transitoire : sur quels éléments 'réels' d'une crise économique et sociale globale se fonde-t-elle ? Pour analyser ce contexte, je me réfère au concept d'une « culture du projet» de Luc Boltanski².

\section{Le contexte social : la précarité et la " culture du projet" (Luc Boltanski)}

Dans les discussions récentes relatives à la crise, on trouve souvent l'idée que ce n'est pas le capitalisme ou le néolibéralisme qui sont en crise, la crise bancaire et économique ayant eu au contraire pour conséquence de renforcer ainsi que d'accélérer la logique néolibérale. Ce système a donc survécu à la crise en y trouvant même un second souffle. Par conséquent, ce sont plutôt les sociétés et les démocraties qui sont toujours en crise $^{3}$ : les gens s'intéressent de moins en moins à la politique ou sympathisent avec des partis ou des mouvements extrémistes, exprimant ainsi leur frustration.

D'un point de vue social, cette crise se manifeste par une augmentation significative de la précarité - dans le monde du travail ainsi que dans la vie privée. Il s'est développé une façon toute nouvelle d'aborder la vie qu'on peut appeler « transitoire » : les gens font face à une précarité ou une insécurité continues, à une instabilité profonde des cadres de vie qui les forcent à improviser, à changer de plus en plus souvent leur façon de vivre, de travailler, de se loger, qui influent sur leur appartenance à un groupe social, voire sur les modèles familiaux. Cette instabilité les oblige en permanence à recommencer à zéro, à s'inventer de nouveau, à

1. - Bernd Stegemann, « Nach der Postdramatik », Theater heute, $\mathrm{n}^{\circ}$ 10/2008, p. 14-21. L'idée d'un néoréalisme théâtral s'est développée dans le contexte des discussions menées autour du concept philosophique d'un Nouveau Réalisme qui fait suite à la pensée postmoderne. Voir Markus Gabriel (éd.), Der Neue Realismus, Frankfurt a.M., Suhrkamp, 2014. C'est surtout dans le monde des arts et de la littérature que ce concept a connu un grand succès.

2. - Boltanski a formulé ce concept à plusieurs reprises. Voir par exemple Luc Boltanski, "Les changements actuels du capitalisme et la culture du projet ", Cosmopolitiques, $\mathrm{n}^{\circ} 12 / 2008$, p. 17-41. Par la suite, les références à cet article sont indiquées entre parenthèses dans le texte.

3. - Cf. Ulrich Karl Preuß, «Social Solidarity and the Crisis of Economic Capitalism. Political Implications », Transit, n 40/2010, p. 105-119. 
s'adapter à des situations diverses, à mener une vie nomade - autant de termes, concepts ou métaphores qui décrivent cette situation du transitoire. C'est cette façon spécifique d'aborder la vie qui se reflète dans les pièces de nos trois dramaturges, ces dernières essayant de trouver une forme esthétique en phase avec cette façon d'être. Leurs pièces présentent de jeunes protagonistes vivant tous dans la précarité : soit dans le monde du travail néolibéral défini par l'idéal de la créativité (Felicia Zeller), soit dans le monde des nouveaux sans-abris victimes de la crise immobilière aux États-Unis (Laura Marks) ou des jeunes nomades d'un monde globalisé qui se retrouvent dans une communauté de hasard dans le Yukon à l'extrémité septentrionale du Canada (Sarah Berthiaume).

Afin de mieux circonscrire la dynamique transitoire et provisoire telle qu'elle est présentée dans ces pièces, on peut recourir au concept de « culture du projet» proposé par Luc Boltanski. Selon lui, cette « culture » est emblématique de la société actuelle et se caractérise avant tout par une activité permanente qui nous mène d'un projet momentané à un autre. La notion de projet, qui trouve son origine dans le monde du travail et notamment celui du management, a peu à peu envahi tous les domaines de notre société, investissant également la vie privée, qu'on songe par exemple au « projet parental» (p. 33) :

L'activité se manifeste dans la multiplicité des projets de tous ordres qui peuvent être menés concurremment ou être développés successivement, le projet constituant, dans cette logique, un dispositif transitoire. La vie est conçue comme une succession de projets, d'autant plus valables qu'ils sont plus différents les uns des autres (p. 24).

La culture du projet définit nos contacts sociaux transitoires dans la mesure où « chacun sait, au moment où il s'engage dans un projet, que l'entreprise à laquelle il va contribuer est destinée à vivre un temps limité, que non seulement elle peut, mais qu'elle doit se terminer» (p. 25). En outre, la culture du projet s'inscrit dans la logique du « réseau » (autre mot clé de l'analyse de Boltanski, p. 24-26) : un grand nombre de projets différents nous met en relation dynamique avec un grand nombre de personnes différentes.

Par conséquent, la principale crainte de chacun - selon Boltanski naît de l'éventualité de perdre la connexion au réseau, de n'avoir plus de projet en vue, d'être mis à l'écart ou même exclu de la culture du projet. A contrario, on fait pleinement partie de ce qu'il appelle la « cité par projet » lorsqu'on est « adaptable et flexible » (p. 25) et qu'on déborde de créativité. Les personnes qui ont du succès sont « des innovateurs qui ont pour modèle les savants et surtout les artistes » (p. 26). C'est ici que l'analyse de Boltanski est révélatrice : le monde des artistes, depuis longtemps un lieu de critique du système, a été lui-même intégré dans la logique néoli- 
bérale. Par conséquent, le monde artistique cherche de plus en plus à se distancier de cette dynamique néolibérale omniprésente. La discussion actuelle autour d'un néoréalisme dans les arts s'inscrit dans ce contexte.

En Allemagne, Bernd Stegemann, professeur des arts de la scène et membre de la Schaubühne Berlin, a à plusieurs reprises, critiqué le théâtre postmoderne ou postdramatique pour sa propension à corroborer le système. Dans son Éloge du réalisme ${ }^{4}$, Stegemann explique que la réalité de la société doit retourner sur les scènes du théâtre et qu'il faut en finir avec les jeux autoréférentiels de l'art postmoderne. Mais à quoi peut bien ressembler cette nouvelle référence à la réalité ? C'est ce que montrent les trois pièces abordées ici.

\section{Démasquer la réalité d'une 'culture du projet' : $\mathrm{X}$-Freunde de Felicia Zeller}

Dans la pièce $X$-Freunde 5 [X-amis] de Felicia Zeller, distinguée comme meilleure pièce germanophone de l'année 2013 par le journal Theater heute, la culture du projet se manifeste à plusieurs niveaux, le titre reflétant déjà la logique du réseau. Vingt-trois petites scènes présentent des situations typiques de trois personnages de la génération des trentenaires : ils ont des professions caractéristiques de cette génération et de son style de vie. Anne, consultante, vient d'ouvrir sa propre agence. Elle travaille sans cesse, conduisant un projet après l'autre : « Je suis par principe occupée et chargée de plusieurs projets en même temps $»^{6}$. En revanche, son mari Holger, traiteur, a dû fermer son entreprise et est à la recherche d'un nouveau projet. En attendant, il s'occupe de la maison, du « projet 'armoire de toilette' » par exemple : «Dès que j'en ai terminé avec le balcon, j'entame le projet 'armoire de toilette' $\gg$. Enfin, il y a Peter, artiste sculpteur en mal d'inspiration. Il doit achever sa série de sculptures intitulée «X-Freunde». Mais au lieu de créer une œuvre d'art intitulée « Le dernier ami » («Der letzte Freund», p. 13) censée répondre aux exigences du marché de l'art et à son impératif de l'originalité, il réfléchit aux raisons qui l'empêchent de se mettre au travail. Il s'incline devant la nouvelle génération de créateurs actifs dans d'autres domaines, galeristes commerciaux et consultants de tout poil en tête.

4. - Bernd Stegemann, Lob des Realismus, Berlin, Verlag Theater der Zeit, 2015.

5. - Felicia Zeller, X-Freunde, Berlin, henschel SCHAUSPIEL Theaterverlag, 2012. La reproduction des passages du texte a été aimablement autorisée par l'éditeur. Par la suite, les références à cette pièce sont indiquées entre parenthèses dans le texte.

6. - « Ich bin grundsätzlich beschäftigt und betreue viele Projekte gleichzeitig. » (p. 43). Nous traduisons.

7. - «Wenn ich mit dem Balkon fertig bin, will ich das Projekt 'Badezimmerschrank' angehen. » (p. 36). 
L'idée de flexibilité, de dynamique permanente se manifeste à tous les niveaux; on trouve des variations sur le thème de la nouveauté et du renouvellement. Ainsi, la première scène s'intitule " nouveau départ » ( Neuanfang », p. 5), la cinquième « réouverture » ("Neueröffnung », p. 19). Les didascalies mentionnent quant à elles un "redémarrage » (« NEUSTART », p. 3) qui fait penser au redémarrage d'un ordinateur. Il suffit d'appuyer sur une touche de clavier ("Tastengriff», p. 3) pour que les personnages continuent à parler de la même manière : " Sans montrer aucun signe de fatigue, les acteurs se remettent tout simplement à parler. $\rangle^{8}$. Les cascades verbales qui expriment - au niveau de la forme - l'épuisement de ces personnages presque toujours à bout de souffle font penser aux Sprachflächen d'Elfriede Jelinek.

L'idée d'une activité continuelle et permanente se traduit enfin dans une « réalisation gymnastique du texte $»^{9}$ montrant la nervosité des personnages, leur instabilité intérieure et extérieure, mentale et corporelle. Les acteurs, tenus de bouger sans cesse, ne restent jamais longtemps dans la même position, exprimant par là leur obsession de toujours être connectés au réseau : " être assis, allongé, debout : aucune position ne dure longtemps. S'allonger en étant assis. S'asseoir en étant debout. Se lever en étant allongé. Tellement de choses à faire. Les acteurs sont toujours joignables $»^{10}$. La succession effrénée de ces scènes brèves donne l'impression que les personnages sont traqués. La pièce ne présente pas d'intrigue dans un sens traditionnel, mais plutôt un état de menace permanente.

Visiblement, la stratégie esthétique de Felicia Zeller consiste à se servir de la logique capitaliste ou néolibérale - de sa dynamique du transitoire et de son vocabulaire du projet - afin de la démasquer au moyen de la satire. Du point de vue du processus de création artistique, cela lui permet d'utiliser des éléments de la réalité du langage comme matériau, puis de travailler ce matériau : sa méthode de transformation est un travail sur la langue comparable à celui de Heiner Müller ou d'Elfriede Jelinek. Il s'agit d'une critique artistique du système par le biais d'une critique du langage. La fragmentation du langage lui permet de mettre l'accent sur la déformation de celui-ci qui est elle-même le signe d'une déformation plus profonde à l'intérieur des personnages. La réplique d'Anne, quand son mari lui conseille de faire une pause, montre à quel point elle a intériorisé la logique capitaliste :

8. — «Die Darsteller setzen ohne Anzeichen von Ermattung einfach wieder an zu sprechen. » (p. 3).

9. — « GYMNASTISCHE TEXTUMSETZUNG», ibid.

10. — «Sitzen, liegen, stehen: Keine Haltung ist lange haltbar. Im Sitzen liegen. Im Stehen sitzen. Im Liegen stehen. So viel zu tun. Die Darsteller sind immer erreichbar. ", ibid. 
Allons, Holger, tu parles avec moi CE N'EST PAS UNE PUB ! Parfois tu parles avec moi comme si j'étais FAIS UNE PAUSE ! Mais je ne suis pas une barre de chocolat! Dans ma branche, on ne peut pas juste FAIRE UNE PAUSE on ne peut pas vendre de l'engagement, de l'implication, du MONDE-EN-PLUS ${ }^{11}$ en travaillant soi-même juste de temps en temps, disons le mardi ou le jeudi $[\ldots]^{12}$.

Parce qu'elle se limite à considérer la surface du langage - qu'elle associe quasi automatiquement à un slogan publicitaire - Anne n'appréhende pas le fait qu'elle a intériorisé l'impératif néolibéral de l'engagement bien qu'il soit dépourvu de tout contenu.

\section{Réalisme symbolique du transitoire : Bethany de Laura Marks}

La pièce Bethany ${ }^{13}$ de la jeune dramaturge américaine Laura Marks, représentée pour la première fois à New York au City Center Stage II, Off-Broadway en 2012, a été montée en 2014/2015 au Nationaltheater à Mannheim. La pièce se déroule en pleine crise immobilière aux ÉtatsUnis et raconte plusieurs jours de la vie d'une jeune femme qui vient d'être expulsée de son logement. Elle s'installe dans un hôtel particulier abandonné dans un quartier bourgeois d'une grande ville américaine. À première vue, son histoire est présentée à la manière d'un well made play anglophone : les dialogues sont écrits dans un anglais américain contemporain, parfois argotique. L'intrigue se déroule en douze scènes. Quand Chrystal, la jeune femme, s'installe dans cette maison abandonnée, elle y rencontre un autre personnage échoué, Gary, un jeune chômeur marginalisé. Ce dernier souffre de fortes attaques de panique et ne peut pratiquement jamais sortir de la maison. Chrystal, qui travaille comme vendeuse automobile dans une agence Saturn, essaie par tous les moyens de maintenir une apparence de vie bourgeoise. Au cours de la pièce, on comprend pourquoi : la perte de son logement a conduit à ce que lui soit retirée la garde de sa fille de cinq ans. Pour convaincre l'assistante sociale qui lui rend visite régulièrement, elle fait semblant de mener une vie réglée afin de récupérer sa fille. Elle est prête à tout

11. - Dans l'original, le néologisme « MEHRWELT » est une paronomasie de «Mehrwert» (valeur ajoutée).

12. - « Also echt, Holger, du redest mit mir DAS IST KEINE WERBUNG! Manchmal redest du mit mir, als wäre ich MACH DOCH MAL PAUSE! Ich bin doch kein Schokoriegel! Du kannst in dieser Branche nicht einfach mal PAUSE du kannst nicht Engagement verkaufen, Einsatz, MEHRWELT und dann selber nur so ein bisschen, vielleicht mal dienstags oder donnerstags [...] » (p. 24).

13. - Laura Marks, Bethany, in : American Next Wave. Four Contemporary Plays from High Tide Festival Theatre, London, Methuen Drama, 2012, p. 163-217. 
pour parvenir à ses fins : contrefaire un bail, se livrer au racket et même commettre un meurtre, celui de Gary, son colocataire de fortune.

L'idée qui sous-tend la pièce et que met en évidence son réalisme social est que ce sont les circonstances de la vie qui déterminent le degré de conscience morale. L'héroïne apparaît comme déformée par la précarité, et cette distorsion porte atteinte aux fondements éthiques de la société. À cette strate réaliste d'emblée perceptible se superpose une strate symbolique : la crise immobilière y figure comme le symbole d'une crise d'identité profonde à l'œuvre dans les sociétés néolibérales. Au sein de la « cité par projet », affirme Boltanski, l'idéal de la mobilité accentue les moments de passage :

Étant donné l'importance accordée à la mobilité, on comprendra facilement que l'une des épreuves sur lesquelles il est mis particulièrement l'accent est constituée par le moment de passage d'un projet à un autre. C'est en effet dans ces moments de changements que se révèle la grandeur des personnes ${ }^{14}$.

Mais la flexibilité et la mobilité causent aussi de nouvelles inquiétudes : les gens sont non seulement envahis par la peur d'être mis à l'écart, mais aussi par

[...] l'angoisse de se perdre dans la multiplicité des activités ; par la peur de voir se dissiper l'unité d'une vie personnelle (comme en témoigne, par exemple, le développement récent des névroses dites « de personnalités multiples ») et par là, d'une certaine façon, de ne plus exister, pour les autres et pour soi-même, en tant que point de référence stable $[\ldots]^{15}$.

Faire construire une maison pour y habiter toute sa vie, voire la transmettre à ses descendants, peut, dans ce contexte, représenter le symbole d'une stabilité obsolète. La crise immobilière reflète, au sens premier du terme, la perte de stabilité. Si un déménagement peut refléter la flexibilité et la dynamique d'une vie réussie, la perte du logement - qui réduit à plus ou moins long terme les gens à l'état de sans-abri - conduit à une véritable crise de l'identité : la perte d'un point stable (extérieur) correspond - comme le suggère cette pièce - à la perte d'un point de référence intérieur, à savoir à l'instabilité d'une identité aux conséquences catastrophiques. Le 'projet' de famille (un projet à long terme) qui prévoit de voir grandir une nouvelle génération, figurée ici par la petite fille de cinq ans, échoue lamentablement. Le prénom de la fille, Bethany, dont la présence sur scène est assurée par un petit portrait photographique,

14. — Luc Boltanski, « Les changements actuels... », op. cit., p. 27.

15. - Ibid., p. $27 s q$. 
a une portée symbolique : il évoque un lieu biblique, la ville natale du pauvre Lazare. Dans la pièce de Laura Marks, il semble figurer l'espoir d'un avenir. Mais la fin tragique montre des personnages dépourvus d'avenir, occupés qu'ils sont à se battre pour survivre au quotidien. Le refus de Chrystal de s'enfuir avec Gary et de commencer une nouvelle vie ailleurs avec lui provoque la colère de ce dernier. Lors d'une dispute, elle lui répond : " Gary, je t'en prie ! Je ne peux pas penser à l'avenir ; je suis obligée de me concentrer sur le présent $»^{16}$.

Lue dans le contexte d'un néoréalisme dramatique, la pièce de Laura Marks montre un retour aux conflits dramatiques : elle met en scène une héroïne tragique plongée dans les affres d'une crise personnelle. En montrant ses personnages dans un contexte social réel et symbolique à la fois, Marks présente une tragédie de nos jours.

\section{Le réalisme des choses passagères : Yukonstyle de Sarah Berthiaume}

La pièce Yukonstyle ${ }^{17}$ de la jeune dramaturge québécoise Sarah Berthiaume, montée simultanément en 2013 au Théâtre d'Aujourd'hui à Montréal et au Théâtre national de la Colline à Paris, est comparable à Bethany. Comme Marks, Berthiaume met des personnages dramatiques au centre de sa pièce. En quatorze scènes, précédées d'une ouverture et ouvrant sur un épilogue, cette pièce donne à voir une communauté de hasard : trois jeunes gens, colocataires pour un temps, habitent un appartement à White Horse, capitale de la province du Yukon à l'extrême nord du Canada. C'est un territoire reculé, un lieu de repli loin des grandes métropoles du monde globalisé. Le lieu est caractérisé par l'immensité de son paysage, par un hiver semblant éternel, par son passé de ruée vers l'or, par sa population autochtone et les animaux sauvages qui l'habitent, tels ces immenses corbeaux survolant la scène.

C'est avant tout l'atmosphère de ce lieu que Berthiaume évoque dans sa pièce. Elle y rassemble des personnages nomades d'aujourd'hui : il y a Yuko, une Japonaise exilée au Yukon parce qu'elle a « checké sur Internet la place au monde où c'est qu'il y avait le moins de Japonais. C'était ici. Faque j'ai tout vendu. Pis je suis venue. » (p. 14) Cette réplique illustre bien la langue stylisée de la pièce : dans sa préface, Berthiaume la caractérise comme " une langue québécoise, mais avec un rythme près de l'anglais. » (p. 7) Le colocataire de Yuko, Garin, est un métis,

16. — « Gary, please! I can't think about the future; I just need to focus on right now. » L. Marks, Bethany, op. cit., p. 212. Nous traduisons.

17. - Sarah Berthiaume, Yukonstyle, Montreuil, Éditions Théâtrales, « En scène », 2013. Par la suite, les références à cette pièce sont indiquées entre parenthèses dans le texte. 
fils de « Dad's » et d'une femme autochtone. Déraciné, Garin cherche la vérité sur sa mère qui a disparu dans les années 1980. Enfin, il y a Kate, une jeune fille enceinte qui a fait une fugue et traverse le Canada en autocar : elle est habillée à la mode « Harajuku» (p. 8), un style de poupée trash d'un quartier de Tokyo, référence ignorée d'elle. Son style représente l'idée de transculturalité et de nomadisme omniprésente dans la pièce, à travers d'une part le métis déraciné avec son " T-shirt des Canucks » (p. 19) qui plonge parfois dans une temporalité indienne, la Japonaise exilée qui fait des « sushis en jambon » au restaurant « High Country Inn » (p. 18) d'autre part et pour finir, la jeune Canadienne habillée comme une Japonaise branchée qui n'en fait qu'à sa tête.

Ce sont des personnages déstabilisés en quête d'un destin meilleur. Divers éléments concourent à créer une ambiance mystérieuse : le lieu, réel et symbolique à la fois, « larger than life », comme l'énonce le slogan officiel du Yukon, des morceaux de musique mélancoliques (à savoir, des chansons des Moldy Peaches et de Neil Young) mais surtout la langue. D’un côté, il y a la " rudesse des dialogues et la pauvreté de la langue des personnages », et en « contrepoids », il y a des «passages narratifs », des « envolées poétiques » (p. 7), comme Berthiaume les appelle, qui se lisent comme de véritables petits poèmes en prose :

Je marche. Je marche jusqu'à plus sentir mes jambes, ma face, mon corps, pis quand je les sens plus, je continue de marcher. Je pense : je veux devenir un bus Greyhound, me faire tatouer des lévriers sur les côtes pis marcher en ligne droite jusqu'à ce que je voie la mer. Je pense : je veux me faire piétiner par un troupeau de bisons, devenir un Mama Burger effouaré, oublié entre deux bancs de neige. Je pense : je veux me gonfler de paysage pis exploser comme une balloune de fête ; devenir une pluie de confettis noirs qui neige, tranquille, sur la nuit cassante du Yukon (p. 40).

Ces passages narratifs ont plusieurs fonctions : ils sont utilisés comme commentaires d'une voix de narrateur qui évoque les lieux et les actions des personnages. Au-delà de ce récit réaliste, les passages narratifs transmettent l'inconscient des personnages dans des moments surréalistes, en expriment leurs rêves et leurs visions.

Contrairement à Laura Marks, Sarah Berthiaume ne raconte pas d'histoire, il n'y a pas d'intrigue mais plutôt une parabole de la vie humaine, état transitoire par excellence : depuis les personnages nomades de passage comme Kate, jusqu'à l'esquisse d'une histoire d'amour entre Garin et Yuko, en passant par les moments clés d'une vie humaine, qu'il s'agisse de la naissance (symbolisée par la grossesse de Kate) ou de la mort (celle du père de Garin).

Le réalisme de cette pièce s'enracine manifestement dans une certaine utopie poétique : le transitoire de la vie, les événements accidentels et 
vite oubliés sont contrebalancés par la densité de la voix poétique. Le passage suivant donne le récit en abrégé, sous forme d'énumération, des semaines que les trois colocataires ont passées ensemble :

Yuko, KaTE et GARIN. - (ensemble ou non) Deux bières tablette, un bois en arrière, trois pieds de neige, du kraft dinner au thon avec des épices à steak, une grippe, la toilette qui flushe plus, une soirée karaoké, un deux pour un sur la Yukon Gold en bouteille, une vieille toune d'Alannah Myles, un lendemain de brosse, des pantoufles, une télé 40 pouces, une game de poker en reprise, une coupure à la main en hachant du céleri, deux points de suture [...] (p. 31).

Cette énumération - une nouvelle fois en forme de poème en prose cherche apparemment à capter des moments de 'vraie vie', des petits bouts de 'réalité, évoquant un tableau poétique composé de petites choses, de détails et de moments fugaces.

Les trois analyses auxquelles nous venons de nous livrer ont pu mettre en évidence trois démarches différentes relevant d'une esthétique du transitoire face à la crise: Felicia Zeller examine la réalité du langage contemporain afin de démasquer la réalité sociale et son impératif du transitoire; Laura Marks se mesure à la réalité de la crise immobilière pour montrer au-delà du simple réalisme social une déstabilisation profonde qui ébranle les fondements moraux d'une société ; tandis que Sarah Berthiaume, en évoquant la réalité des petites choses éphémères, essaie de créer un 'contrepoids' poétique dans un monde globalisé peuplé de nouveaux nomades. 